\title{
ANALYSIS OF STRAIGHTENING FORMULA
}

\author{
DEVADATTA M. KULKARNI \\ Department of Mathematics \\ Oakland University \\ Rochester Michigan 48063.
}

(Received March 23, 1987)

\begin{abstract}
The straightening formula has been an essential part of a proof showing that the set of standard bitableaux (or the set of standard monomials in minors) gives a free basis for a polynomial ring in a matrix of indeterminates over a field .The straightening formula expresses a nonstandard bitableau as an integral linear cobmbination of standard bitableaux. In this paper we analyse the exchanges in the process of straightening a nonstandard pure tableau of depth two. We give precisely the number of steps required to straighten a given violation of a nonstandard tableau. We also characterise the violation which is eliminated in a single step.
\end{abstract}

Keywords and Phrases. Bitableaux, standard bitableaux, unitableaux of pure length and depth two, monomials in minors, Straightening formula, oddity function, violation, good violation, number of steps to straighten a nonstandard unitableau .

AMS Primary Classification: 15,05 AMS Secondary Classification: 13

\section{INTRODUCTION.}

The straightening formula has been an intregral part of the theorem showing that the set of all standard monomials in minors of a matrix of indeterminates form a free basis of the polynomial ring in those indeterminates. It tranforms a nonstandard bitableau into an integral linear combination of the standard bitableaux, which makes sense only after using a correspondence between bitableaux and monomials in minors. The straightening formula is given in Rota-Doubillet -Stein [1] first and given again and exploited greatly in Desarmenien-Kung-Rota [2], DeConcini-Procesi [3], DeConcini Eisenbud - Procesi [4]. Abhyankar [5] gives a proof of the above mentioned theorem by explicitly counting the dimension of a vector space generated by all the standard bitableaux of area $\mathrm{V}$ and length less than or equal to $\mathrm{p}$, and deduces the result that the ideal generated by the $\mathrm{p}$ by $\mathrm{p}$ minors of a matrix of indeterminates is Hilbertian. In this exposition one also finds a form of the straightening formula which is very amenable for analysis. In a sequel of [5], Abhyankar has proved the Hilbertianness of a much more general determinantal ideal following the same strategy of counting and straightening, eliminating the proof of linear independence of standard bitableaux [6]. He also states the straightening formula in much general form and proposes the

Problem: Given a nonstandard bitableau $\mathrm{T}$, if $\mathrm{T}=\sum \mathrm{c}_{1} \mathrm{~T}_{\mathrm{i}}$ is the expression for $\mathrm{T}$ given by the Straightening formula where $T_{i}$ 's are standard bitableaux; can one determine $c_{1}$ 's in terms of $T$ ? 
He defines the final integer function there which helps to give the coefficients $c_{1}$. He also gives a recursion satisfied by this fin function, and states a problem of finding fin in terms of a given nonstandard bitableau. More knowledge about the number of steps required to straighten a given nonstandard bitableau will help finding this fin function.

In this paper we analyse the formula as given in [5]. For an analysis of the straightening formula for a nonstandard unitableau it is enough to look at a nonstandard pure unitableau of depth two. We state a form of the straightening formula using an arbitrary violation. The proof of this form is identical to the proof in [5]. We give an exact number of steps required to eliminate the violation from all unitableaux obtained in the straightening. As a part of our proof we give a detailed analysis of exchanges in the straightening and characterise a violation which gets eliminated in a single step (a good violation).

\section{NOTATION AND TERMINOLOGY.}

Let $\left[X_{i j}\right]_{1 \leq i \leq m, 1 \leq j \leq n}$ be a matrix all of whose entries are indeterminates over a field $K$. Let $Y$ be an $m$ by $m+n$ matrix formed by keeping the first $m$ columns of $Y$ to be those of $X$ and putting the $(n+i) t h$ column to be $(m-i+1)$ th column of an $m$ by $m$ identity matrix for $1 \leq \mathrm{i} \leq \mathrm{m}$. Throughout the discussion we use the word "minor " with the meaning as "determinant of a minor". In the proof of a theorem, showing the set of standard monomials in maximal size minors of $Y$ to be a free basis of K[Y], the spanning part of it is done by repeated applications of the straightening formula to a nonstandard unitableau of pure length $\mathrm{m}$ and bounded by $\mathrm{m}+\mathrm{n}$. Using this, one proves that the standard monomials in minors of $\mathrm{X}$ form a free basis of $\mathrm{K}[\mathrm{X}]$ by invoking the correspondence between all minors of $\mathrm{X}$ and the maximal size minors of $\mathrm{Y}$, and then the correspondence between tableaux and monomials in minors of $\mathrm{X}$.

A univector of length $m$ and bounded by $p$ is an increasing sequence of $m$ positive integers which are bounded by $p$. To a univector of length $m$ and bounded by $p$ there corresponds an $m$ by $m$ ( $a$ maximal size) minor of an $m$ by $p$ matrix of indeterminates which is formed by picking up the corresponding $m$ columns. A unitableau of depth $d$ is a sequence of $d$ univectors, written as $A(1) A(2) \ldots . A(d)$. By a pure unitableau of length $m$ and bounded by $p$ we mean a unitabeau each constituent univector has length $\mathrm{m}$ and is bounded by $\mathrm{p}$. Given two univectors

$$
A=\left(A_{1}<A_{2}<\ldots<A_{p}\right) \text { and } B=\left(B_{1}<B_{2}<\ldots<B_{q}\right)
$$

we say that

$$
\mathrm{A} \leq \mathrm{B} \text { if } \mathrm{p} \geq \mathrm{q} \text { and } \mathrm{A}_{\mathrm{i}} \leq \mathrm{B}_{\mathrm{i}} \text { for } 1 \leq \mathrm{i} \leq \mathrm{q}
$$

A unitableau $A(1) A(2) \ldots A(d)$ is standard if $A(i) \leq A(i+1)$ for $1 \leq i \leq d-1$. A monomial in maximal size minors of an $\mathrm{m}$ by $\mathrm{p}$ matrix of indeterminates corresponding to a standard pure unitableau of length $\mathrm{m}$ and bounded by $\mathrm{p}$ is said to be standard.

For analysis one has to concentrate on unitableaux of pure length $m$ and depth two. Let all unitableaux be bounded by $\mathrm{p}$ hereonwards. Let mom $(\mathrm{X}, \mathrm{AB})$ be a monomial in maximal size minors of an $m$ by $p$ matrix $X$ of indeterminates over a field $K$. Given a unitableau $A B$ of pure length $m$ and depth two as

$$
\begin{aligned}
& \mathrm{A}_{1}<\mathrm{A}_{2}<\ldots<\mathrm{A}_{\mathrm{m}} \\
& \mathrm{B}_{1}<\mathrm{B}_{2}<\ldots<\mathrm{B}_{\mathrm{m}},
\end{aligned}
$$


we say that the $i$-th column is straight if $A_{i} \leq B_{i}$ and the $i$-th column is a violation if $A_{i}>B_{i}$ and we define the violation set as

$$
V(A B)=\left\{i: 1 \leq i \leq m, A_{i}>B_{i}\right\}
$$

and the oddity function for $\mathrm{AB}$ by putting for each $\mathrm{i}$ with $1 \leq \mathrm{i} \leq \mathrm{m}$,

$$
\mathrm{N}[\mathrm{AB}](\mathrm{i})=\operatorname{card}\left(\left\{\mathrm{k}: 0 \leq \mathrm{k} \leq \mathrm{i}-1, \mathrm{~A}_{\mathrm{i}-\mathrm{k}}>\mathrm{B}_{\mathrm{i}}+\mathrm{k}\right\}\right) .
$$

We note that $A B$ is standard if $V(A B)=\emptyset$ and if $N[A B](i)=0$ for $1 \leq i \leq m$. We say that the $v$-th column is good if $v \varepsilon V(A B)$ and $N[A B](v)=1$.

\section{STRAIGHTENING FORMULA.}

We are giving here the straightening formula in [5] using any violation.

THEOREM 1: Let $\mathrm{AB}$ be a nonstandard pure unitableau of length $\mathrm{m}$ and depth two and bounded by $\mathrm{p}$. Let $v \varepsilon \mathrm{V}(\mathrm{AB})$, and let

$$
\underline{A}[v]=\left\{A_{k}: v \leq k \leq m\right\} \text { and } \underline{B}[v]=\left\{B_{k}: 1 \leq k \leq v\right\} \text {. }
$$

We form a set $\underline{E}[v]$ as

$$
\begin{gathered}
\underline{E}[v]=\{(a, b): a \subset \underline{A}[v], b \subset \underline{B}[v],(A \backslash a) \cap b=\emptyset=(B \backslash b) \cap a \text { and } \\
\operatorname{card}(a)=\operatorname{card}(b) \neq 0\}
\end{gathered}
$$

For $\mathrm{E}=(\mathrm{a}, \mathrm{b}) \varepsilon E[\mathrm{v}]$, let sat $(\mathrm{A}, \mathrm{E})$ and sat $(\mathrm{B}, \mathrm{E})$ denote the univectors of length $\mathrm{m}$ formed by sets respectively $(A \backslash a) \cup b$ and $(B \backslash b) \cup a$, and sat $[A B, E]$ denote a satellite unitableau corresponding to $\mathrm{E} \varepsilon \mathrm{E}[\mathrm{v}]$ written as

$$
\text { sat }[A B, E]=\operatorname{sat}(A, E) \text { sat }(B, E) \text {. }
$$

We have

$$
\operatorname{mom}(\mathrm{X}, \mathrm{AB})=\sum_{\mathrm{E} \varepsilon \underline{\mathrm{E}[\mathrm{v}]}} \#(\mathrm{AB}, \mathrm{E}) \operatorname{mom}(\mathrm{X}, \operatorname{sat}[\mathrm{AB}, \mathrm{E}])
$$

where \# $(\mathrm{AB}, \mathrm{E})$ is + or - according to the sign in the Laplace development and the signs required to form sat $(A, E)$ and sat ( B , E ) for all E $\varepsilon \underline{E}[v]$.

PROOF. The proof of this theorem is the same as that in [5] which is done for $v=\min V(A B)$ there.

We say that a pure unitableau $A * B *$ of length $m$ occurs in the first step of straightening of $A B$ with respect to $\mathrm{v}$ if

$\mathrm{v} \varepsilon \mathrm{V}(\mathrm{AB})$ and $\mathrm{A}^{*}=$ sat $(\mathrm{A}, \mathrm{E})$ and $\mathrm{B}^{*}=$ sat $(\mathrm{B}, \mathrm{E})$ for some $\mathrm{E} \varepsilon \underline{\mathrm{E}}[\mathrm{v}]$, 
we write it as $A^{*} B^{*} \varepsilon S[A B, v, 1]$. We say that $A^{* *} B^{* *}$ occurs in the second step of the straightening of $A B$ with respect to $v$ if

$$
\mathrm{v} \varepsilon \mathrm{V}\left(\mathrm{A}^{*} \mathrm{~B} *\right) \text { and } \mathrm{A}^{* *} \mathrm{~B}^{* *} \varepsilon \mathrm{S}\left[\mathrm{A}^{*} \mathrm{~B}^{*}, \mathrm{v}, 1\right]
$$

for some $A^{*} B^{*} \varepsilon S[A B, v, 1]$ and we write it as $A^{* *} B^{* *} \varepsilon S[A B, v, 2] ;$ and we may define $S[A B, v, s]$ for $s \geq 1$ inductively.

\section{ANALYSIS OF EXCHANGES.}

In this section let $A B$ be as given above and $v \varepsilon V(A B)$ and $A^{*} B^{*} \varepsilon S[A B, v, 1]$ for $(a, b) \varepsilon E[v]$ as described in the Theorem 1. Let $N[A B](v)=q+1$ and card $(a)=\operatorname{card}(b)=r$. We note the condition

$$
\text { (A } \backslash a) \cap b=\varnothing=(B \backslash b) \cap a
$$

which we are going to use strongly .

THEOREM 2: If $\operatorname{card}(\mathrm{a})=\operatorname{card}(\mathrm{b})=\mathrm{r} \geq \mathrm{q}+1$,

$$
v \varepsilon V(A * B *) \text { for all } A * B * \varepsilon S[A B, v, 1]
$$

PROOF: Since every element of $\left\{\mathrm{A}_{1}, \mathrm{~A}_{2}, \ldots, \mathrm{A}_{\mathrm{v}}-\mathrm{q}-1\right\} \cup \mathrm{b}$ is smaller than every element of $\left\{A_{V-q}, A_{V}-q+1, \ldots, A_{m}\right\} \backslash a$, and by (4.0) and the given,

$$
\operatorname{card}\left(\left\{A_{1}, A_{2}, \ldots, A_{v}-q-1\right\} \cup b\right)=v-q-1+r \geq v,
$$

we have $B^{*}{ }_{v} \varepsilon\left\{A_{1}, A_{2}, \ldots, A_{v}-q-1\right\} \cup b$.

Since every element of $\left\{B_{v}+q+1, B_{v}+q+2, \ldots, B_{m}\right\} \cup$ a is greater than every element of $\left\{B_{1}, B_{2}, \ldots, B_{v}+q\right\} \backslash b$, and by (4.0) and the given,

$$
\operatorname{card}\left(\left\{B_{v}+q+1, B_{v}+q+2, \ldots, B_{m}\right\} \cup a\right)=m-v-q+r \geq m-v+1,
$$

we have $A^{*}{ }_{v} \varepsilon\left\{B_{v}+q+1, B_{v}+q+2, \ldots, B_{m}\right\} \cup a$.

By noting that every element of $\left\{A_{1}, A_{2}, \ldots, A_{v-q-1}\right\} \cup b$ is smaller than or equal to every element of $\left\{B_{v}+q+1, B_{v}+q+2, \ldots, B_{m}\right\} \cup$ a as $A_{v}-q-1 \leq B_{v}+q+1$ and $A_{v}>B_{v}$, we have $A^{*} v \leq B^{*}{ }_{v}$, and so $v \varepsilon V\left(A^{*} B^{*}\right)$ for all $A^{*} B^{*} \varepsilon S[A B, v, 1]$.

COROLLARY 3: A violation $\mathrm{v}$ in $\mathrm{V}(\mathrm{AB})$ is good if and only if $\mathrm{v} \notin \mathrm{V}\left(\mathrm{A}^{*} \mathrm{~B}^{*}\right)$ for all $A * B^{*} \varepsilon S[A B, v, 1]$ if and only if $A_{v+1} \geq B_{v-1}$.

PROOF: It follows from above theorem by noting that $\mathrm{v}$ is good if $\mathrm{q}=0$ and card $(a)=$ card $(b) \neq 0$ forces $r \geq 1$. The second part follows from the definition of $N[A B](v)$.

LEMMA 4: If $1 \leq$ card $(\mathrm{a})=\operatorname{card}(\mathrm{b})=\mathrm{r} \leq \mathrm{q}, \mathrm{v} \varepsilon \mathrm{V}\left(\mathrm{A}^{*} \mathrm{~B}^{*}\right)$ for those $\mathrm{A}^{*} \mathrm{~B} * \mathrm{~S}[\mathrm{AB}, \mathrm{v}, 1]$.

PROOF: Since every element of $\left\{A_{v}-q, A_{v}-q+1, \ldots, A_{m}\right\} \backslash$ a is greater than every element of $\left\{A_{1}, A_{2}, \ldots, A_{v}-q-1\right\} \cup b$, and by (4.0) and $q-r \geq 0$, 


$$
\operatorname{card}\left(\left\{\mathrm{A}_{1}, \mathrm{~A}_{2}, \ldots, \mathrm{A}_{\mathrm{v}}-\mathrm{q}-1\right\} \cup \mathrm{b}\right)=\mathrm{v}-\mathrm{q}-1+\mathrm{r} \leq \mathrm{v}-1,
$$

we have $A^{*}{ }_{v} \varepsilon\left\{A_{v}-q, A_{v-q}+1, \ldots, A_{m}\right\} \backslash a$.

Since every element of $\left\{B_{1}, B_{2}, \ldots, B_{v+q}\right\} \backslash b$ is smaller than every element of $\left\{B_{v}+q+1, B_{v}+q+2, \ldots, B_{m}\right\} \cup a$, and by (4.0),

$$
\operatorname{card}\left(\left\{B_{1}, B_{2}, \ldots, B_{v}+q\right\} \backslash b\right)=v+q-r \geq v
$$

we have $B *{ }_{v} \varepsilon\left\{B_{1}, B_{2}, \ldots, B_{v}+q\right\} \backslash b$.

By noting that every element of $\left\{A_{v}-q, A_{v}-q+1, \ldots, A_{m}\right\} \backslash a$ is greater than every element of $\left\{B_{1}, B_{2}, \ldots, B_{v}+q\right\} \backslash b$, we have

$$
\mathrm{A}^{*}{ }_{\mathrm{v}}>\mathrm{B}^{*} \mathrm{v},
$$

and so $v \varepsilon V\left(A^{*} B^{*}\right)$ for those $A^{*} B^{*} \varepsilon S[A B, v, 1]$.

THEOREM 5: For all $\mathrm{A}^{*} \mathrm{~B} * \varepsilon \mathrm{S}[\mathrm{AB}, \mathrm{v}, 1]$, for $1 \leq \mathrm{i} \leq \mathrm{m}$,

$$
N[A * B *](i) \leq N[A B](i)
$$

There exists $(a, b)$ in $E[v]$ such that for the corresponding $A^{*} B^{*}$, we have

$$
N[A * B *](v)=N[A B](v)-1 .
$$

PROOF: Putting N[AB] (i) $=\mathrm{n}$ ( $\mathrm{i}$ ) for $1 \leq \mathrm{i} \leq \mathrm{m}$, by the definition of $\mathrm{N}$, we have

$$
\mathrm{A}^{*_{\mathrm{i}}-\mathrm{n}(\mathrm{i})} \leq \mathrm{A}_{\mathrm{i}-\mathrm{n}(\mathrm{i})} \leq \mathrm{B}_{\mathrm{i}+\mathrm{n}(\mathrm{i})} \leq \mathrm{B}^{*}{ }_{\mathrm{i}+\mathrm{n}(\mathrm{i})}
$$

since $A^{*}{ }_{k} \leq A_{k}$ and $B^{*}{ }_{k} \geq B_{k}$ for $1 \leq k \leq m$ as every element in $A$ which is removed is being replaced by a smaller element in $\mathrm{B}$ and every element in $\mathrm{B}$ which is removed is being replaced by a greater element in A. From the inequality it follows that for $1 \leq \mathrm{i} \leq \mathrm{m}$,

$$
N\left[A^{*} B^{*}\right](i) \leq N[A B](i)
$$

To prove the second part we have to first show that $A^{*} v-q \leq B^{*} v+q$ for all $E$ in $\underline{E}[v]$ by letting $n(v)=q+1$. Since every element of $\left\{A_{1}, A_{2}, \ldots, A_{v}-q-1\right\} \cup b$ is smaller than every element of $\left\{A_{V-q}, A_{v-q+1}, \ldots, A_{m}\right\} \backslash a$, and by (4.0) and $r-1 \geq 0$,

$$
\operatorname{card}\left(\left\{A_{1}, A_{2}, \ldots, A_{v}-q-1\right\} \cup b\right)=v-q-1+r \geq v-q,
$$

we have $A^{*}{ }_{v-q} \varepsilon\left\{A_{1}, A_{2}, \ldots, A_{v-q-1}\right\} \cup b$.

Since every element of $\left\{B_{v}+q+1, B_{v}+q+2, \ldots, B_{m}\right\} \cup$ a is greater than every element of $\left\{B_{1}, B_{2}, \ldots, B_{v}+q\right\} \backslash b$, and by (4.0),

$$
\operatorname{card}\left(\left\{B_{v}+q+1, B_{v}+q+2, \ldots, B_{m}\right\} \cup a\right)=m-v-q+r \geq m-v-q+1,
$$


we have $B^{*} v+q \varepsilon\left\{B_{v}+q+1, B_{v}+q+2, \ldots, B_{m}\right\} \cup$ a.

By noting that every element of $\left\{A_{1}, A_{2}, \ldots, A_{V}-q-1\right\} \cup b$ is smaller than or equal to every element of $\left\{B_{v+q+1}, B_{v}+q+2, \ldots, B_{m}\right\} \cup$ a as $A_{v-q}-1 \leq B_{v}+q+1$ and $A_{v}>B_{v}$, we have

$$
A^{*}{ }_{v-q} \leq B^{*}{ }_{v+q}
$$

and so $\mathrm{N}\left[\mathrm{A}^{*} \mathrm{~B}^{*}\right](\mathrm{v})<\mathrm{q}=\mathrm{N}[\mathrm{AB}](\mathrm{v})$.

By putting $a=\left\{A_{u}\right\}$ and $b=\left\{B_{w}\right\}$ where $A_{u}=\min \underline{A}[v] \backslash B$ and $B_{W}=\max \underline{B}[v] \backslash A$, we note that for the corresponding $A^{*} B^{*}$,

$$
A_{k}^{*}=A_{k-1} \quad \text { for } \quad v-q+1 \leq k \leq u
$$

and

$$
\mathrm{B}^{*} \mathrm{k}=\mathrm{B}_{\mathrm{k}+1} \quad \text { for } \quad \mathrm{w} \leq \mathrm{k} \leq \mathrm{v}+\mathrm{q}-1
$$

as

$$
\operatorname{card}\left(\left\{A_{1}, A_{2}, \ldots, A_{v}-q-1\right\} \cup b\right)=v-q
$$

and

$$
\operatorname{card}\left(\left\{B_{v}+q+1, B_{v}+q+2, \ldots, B_{m}\right\} \cup a\right)=m-v-q,
$$

it follows that

$$
A^{*} v-q+1=A_{v}-q>B_{v}+q=B^{*} v+q-1 .
$$

By the definition of $\mathrm{N}$ and knowing that $\mathrm{A}^{*}{ }_{\mathrm{v}-\mathrm{q}} \leq \mathrm{B}^{*} \mathrm{v}+\mathrm{q}$, we have

$$
\mathrm{N}\left[\mathrm{A} * \mathrm{~B}^{*}\right](\mathrm{v})=\mathrm{N}[\mathrm{AB}](\mathrm{v})-1
$$

The existence of $u$ is assured by the definition of $v$ and

$$
\operatorname{card}(\underline{\mathrm{A}}[\mathrm{v}])>\operatorname{card}\left(\left\{\mathrm{B}_{\mathrm{v}+1}, \mathrm{~B}_{\mathrm{v}}+2, \ldots, \mathrm{B}_{\mathrm{m}}\right\}\right) .
$$

The existence of $w$ is assured by the definition of $v$ and

$$
\operatorname{card}(\underline{B}[v])>\operatorname{card}\left(\left\{A_{1}, A_{2}, \ldots, A_{v}-1\right\}\right) .
$$

THEOREM 6: If we apply the straightening formula to $\mathrm{AB}$ repeatedly using $\mathrm{v} \varepsilon \mathrm{V}(\mathrm{AB})$, for all $A^{*} B^{*} \varepsilon S[A B, v, N[A B](v)]$ we have that $v \notin V\left(A^{*} B^{*}\right)$. The number $N[A B](v)$ is the smallest integer $s$ such that for all $A^{*} B^{*} \varepsilon S[A B, v, s]$ we have that $v \notin V\left(A^{*} B^{*}\right)$.

PROOF: It follows immediately from the previous theorem and recalling that

$$
v \notin V\left(A^{*} B^{*}\right) \text { if } N[A B](v)=0
$$

From the above Theorem it is clear that starting with $\mathrm{AB}$ and applying the straightening formula $\mathrm{N}[\mathrm{AB}](\mathrm{v})$ times we can express $\mathrm{AB}$ ( or mom ( $\mathrm{X}, \mathrm{AB}$ )) as an integral linear combination of 
unitableaux which do not have $\mathrm{v}$ in their violation sets. In this process we do not perform any cancellations as we go. With this in mind, talking of the number of steps required to straighten a nonstandard unitableau is not confusing. By the above theorem and noting that the oddity function drops at each step, it follows that a nonstandard unitableau $\mathrm{AB}$ can be straightened in at most $\Sigma_{1 \leq \mathrm{i}} \leq \mathrm{m} \mathrm{N}[\mathrm{AB}]$ ( i ) steps .

\section{ACKNOWLEDGEMENTS.}

I would like to thank Professor Shreeram S. Abhyankar for his continuous encouragement and guidance while doing my Ph. D. thesis with him. I would like to thank the Bhaskaracharya Pratishthana for its hospitality.

\section{REFERENCES.}

[1] Doubillet, Rota, Stein, Foundations of combinatorics IX : Combinatorical methods in invariant Theory; Studies in applied mathematics 53,185-216 (1974).

[2] Desarmenien, Kung, Rota, Invariant theory,Young bitableaux, and combinatorics; Advances in mathematics 22,No.1,63-92 (1978).

[3] DeConcini, Procesi, A characteristic free approach to invariant theory, Advances in mathematics 21,330-354 (1978).

[4] DeConcini, Eisenbud, Procesi,Young diagrams and determinantal varieties; Inventiones mathematicae 56,129-165 (1980).

[5] Abhyankar S.,Combinatorie des tableaux de Young, varietes determinantielles et calcul de fonctions de Hilbert; Nice lecture notes by A. Galligo ; Universita E. Polytecnico di Torino : Rendiconti del Seminario Matematico (1985).

[6] Abhyankar S., Enumerative Combinatorics of Young Tableaux (Forthcoming in the Monograph Series of Marcel Dekkar ) 


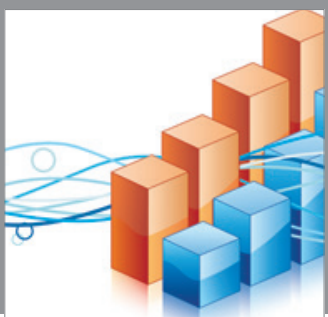

Advances in

Operations Research

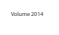

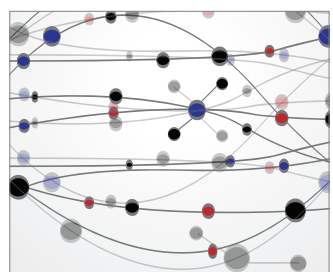

\section{The Scientific} World Journal
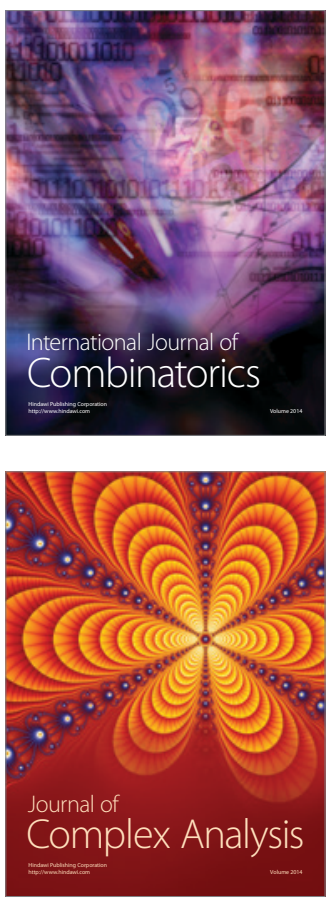

International Journal of

Mathematics and

Mathematical

Sciences
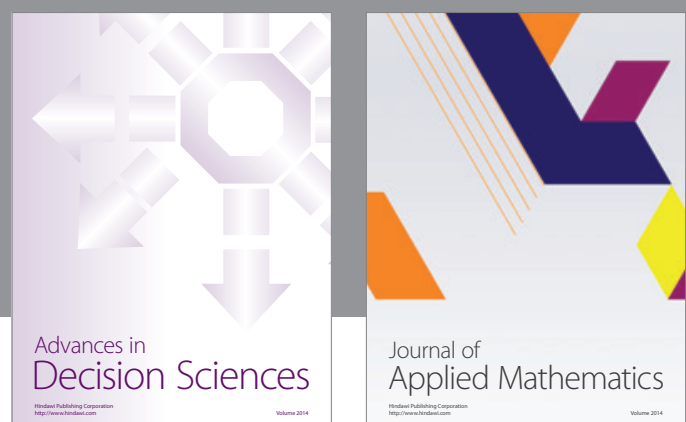

Journal of

Applied Mathematics
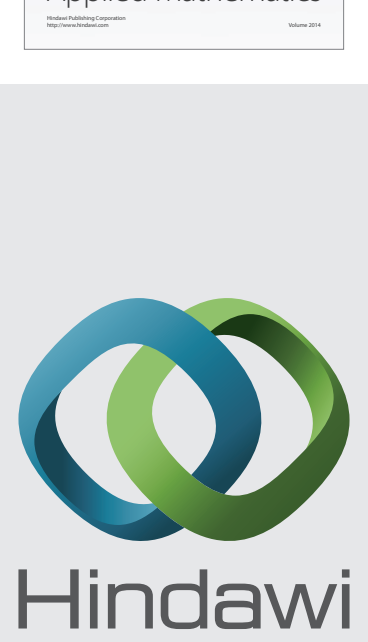

Submit your manuscripts at http://www.hindawi.com
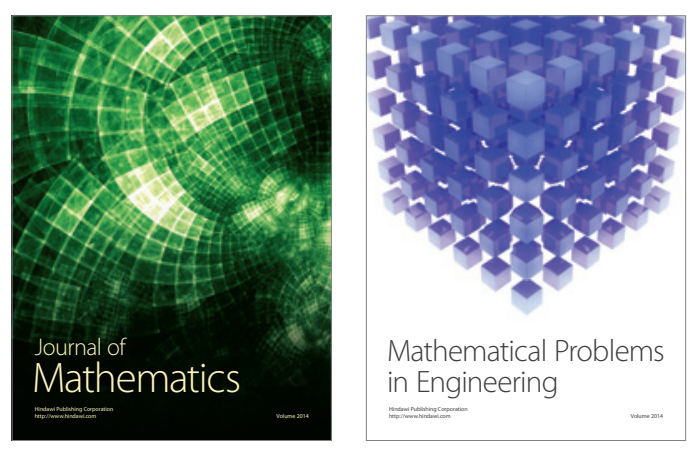

Mathematical Problems in Engineering
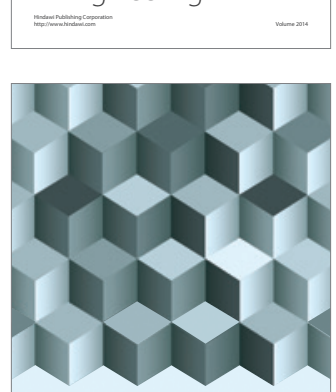

Journal of

Function Spaces
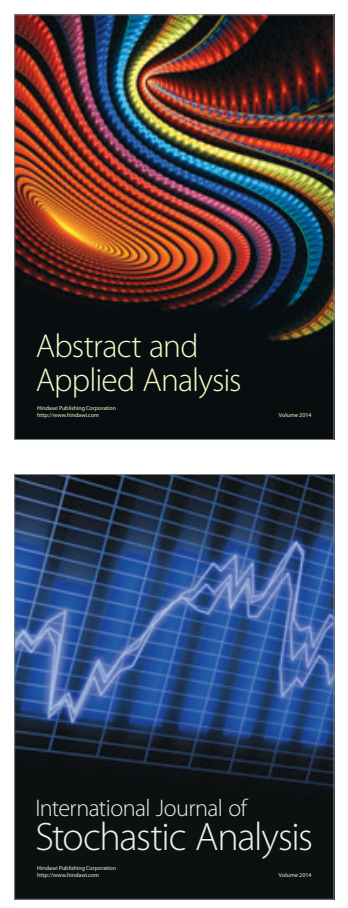

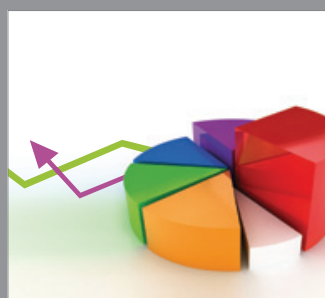

ournal of

Probability and Statistics

Promensencen
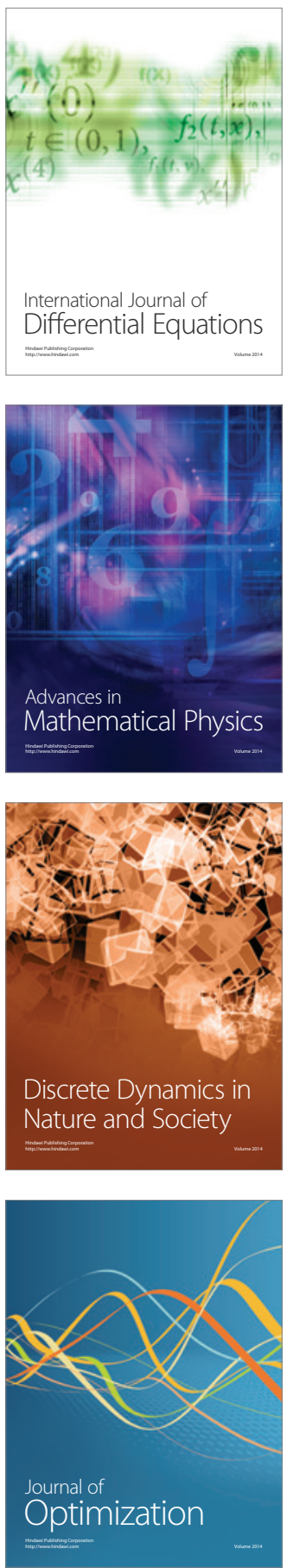